\title{
Técnica de cierre diferido de incisiones de fasciotomías con elásticos*
}

\author{
Dr. LEOPOLDO MARINÉ M. ${ }^{1}$
}

1 Departamento de Cirugía Vascular y Endovascular, Facultad de Medicina, Pontificia Universidad Católica, Santiago, Chile.

\section{Closure of fasciotomy wounds using a vessel loop shoelace}

\section{Introducción}

El síndrome compartamental constituye una urgencia médica, ya que al no ser tratada oportunamente, habitualmente conduce a secuelas neurológicas, amputaciones, insuficiencia renal o muerte. Traumatismos, hemorragias postoperatorias, edema post-reperfusión de isquemia arterial severa o prolongada, entre otros, generan un aumento patológico de la presión de los tejidos contenidos en un compartimento anatómico inexpansible limitado por estructuras óseas y aponeurosis musculares, más frecuentemente en las extremidades inferiores. El aumento progresivo de la presión intracompartamental es responsable de lesiones por compresión de nervios, venas, arterias y fibras musculares, que son responsables de las manifestaciones clínicas que aparecen progresivamente: dolor muy intenso, desproporcionado y progresivo, déficit neurológico sensitivo y luego motor de la extremidad, gran tensión y sensibilidad a la palpación de los compartimentos afectados e isquemia con pérdida de pulsos en casos avanza$\operatorname{dos}^{1}$.

El tratamiento fundamental es descomprimir los compartimentos por medio de fasciotomías. Éstas deben ser amplias, involucrar la totalidad de los compartimentos amenazados y precoces (su postergación determina secuelas irreversibles o amenaza de la viabilidad de la extremidad). Una vez abierta la piel y aponeurosis, es esperable la herniación de los distintos músculos descomprimiendo los compartimentos.

Más de un tercio de los pacientes con fasciotomía presentan complicaciones, la más frecuente y temida es la infección, pudiendo ser determinante en el destino final de la extremidad ${ }^{2-4}$. Contribuyen al desarrollo de infección: la prolongada y amplia exposición de partes blandas favorecida por la retracción progresiva de los bordes cutáneos, la presencia de músculo isquémico o necrótico, y que la indicación de fasciotomía haya sido de origen vascular y en las extremidades inferiores ${ }^{2}$.

El manejo habitual de las heridas asociadas a fasciotomías requiere de injertos dermo-epidérmicos, colgajos fascio-cutáneos y/o el cierre por segunda intención. Una alternativa de manejo, descrita por Harris en $1993^{5}$ y complementada por Berman en $1994^{6}$, es la denominada "shoelace technique" por su semejanza con la disposición de los cordones en los zapatos. Dado que esta técnica pareciera no ser conocida en nuestro medio, me parece interesante comunicarla para difusión a la comunidad quirúrgica local.

*Recibido el 22 de Septiembre de 2008 y aceptado para publicación el 16 de Octubre de 2008.

Correspondencia: Dr. Leopoldo Mariné M.

Apoquindo 3990 Of. 601, Santiago. Fax (562) 6326812

E-mail: marinepolo@yahoo.com 


\section{Técnica}

Objetivo: Crear una malla con elásticos vasculares (vessel loops) de contención, que cumple tres funciones: $1^{\circ}$ mantener a los músculos herniados aislados previniendo infecciones, $2^{\circ}$ evitar la retracción de la piel y $3^{\circ}$ permitir el afrontamiento rápido de los bordes cutáneos en los días posteriores, al tensionar los elásticos y disminuir el área de exposición. Esto se realiza en forma gradual y progresiva en la medida que disminuye el edema muscular.

Requerimientos: cirujano, equipo de curación, elásticos vasculares de silicona (Surg-I-Loop, Scanlan International ${ }^{\circledR}$ Saint Paul, MN, Estados Unidos u otros) y sutura o grapas metálicas (Proximate Manipler AZ-35W, B. Braun ${ }^{\circledR}$, Chasseneuil, Francia u otras).

Técnica: Se comienza en uno de los ángulos de la herida, fijándose a su vértice la zona media del

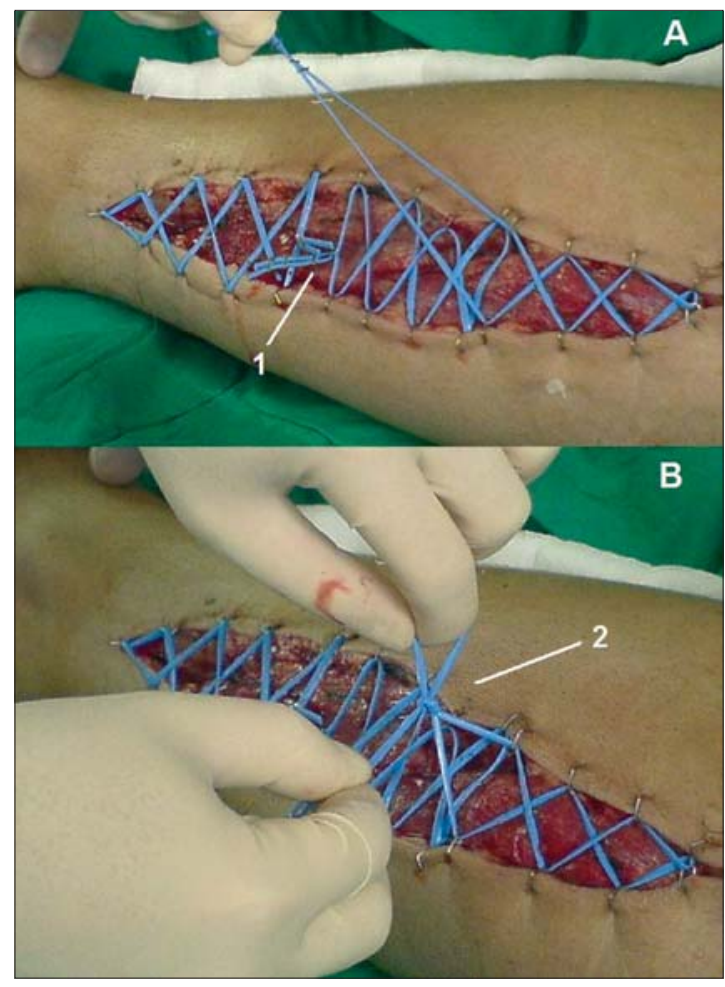

Figura 1. Herida de fasciotomía de pierna derecha. Se observan elásticos vasculares en disposición entrecruzada y fijados por grapas metálicas ("shoelace technique"). 1A línea 1: señala la utilización de clips vasculares para unir ambos extremos de elásticos y dar tensión al los bordes opuestos de la herida. 1B línea 2: señala la alternativa de unión de extremos de elásticos utilizando nudos. En ambos casos los elásticos quedan unidos en el centro de la herida. largo del elástico con dos grapas. Posteriormente, se procede a fijar interrumpidamente el elástico al borde de piel con la colocación de múltiples grapas, en sentido centrípeto al centro de la herida, en dirección diagonal desde un borde de la piel al contrario, a intervalos de 1,5 a $2 \mathrm{~cm}$.

Se avanza en ambos extremos del elástico simultáneamente, siguiendo así una trayectoria zigzagueante, de similar forma a como se colocan los cordones de los zapatos, para finalmente configurar una malla de enrejado similar a la de la Figura 1.

Una vez próximo a finalizar el largo del elástico se procede a colocar clips vasculares (Figura 1 A-1) o a realizar nudos a los remanentes de los elásticos (Figura 1 B-2), de manera que el clip o el nudo quede ubicado en el centro de la herida, equidistante
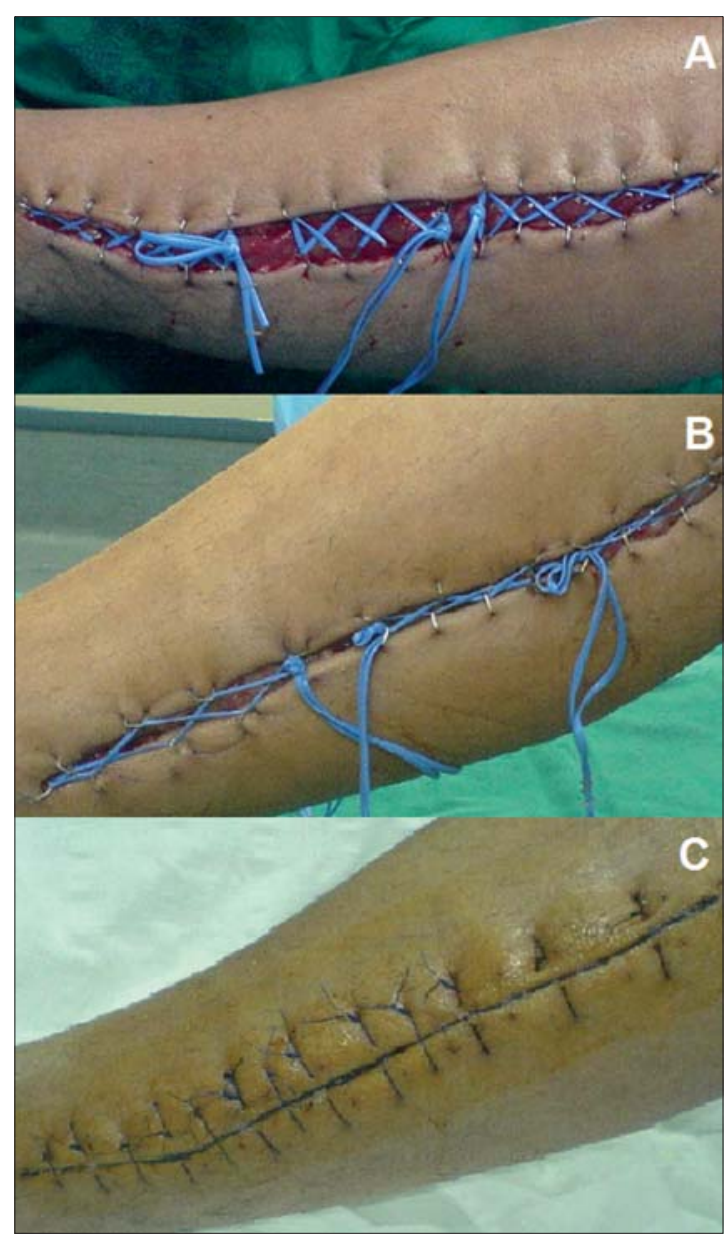

Figura 2. Herida de fasciotomía en distintos momentos de cierre: $\mathbf{2 A}$ a los 3 días. $\mathbf{2 B}$ a los 7 días y en $\mathbf{2 C}$ a los 9 días es posible el cierre de la herida con puntos no reabsorbibles. 
de ambos bordes, y con una tensión suave mantenida. Posteriormente, se repite igual técnica comenzando desde el otro ángulo de la herida. Ante extensas fasciotomías y gran herniación de músculo, serán necesarios elásticos intermedios adicionales.

El momento de la aplicación de esta técnica de cierre es al final de la fasciotomía, inicialmente sin aplicación de tensión. En los días posteriores se efectuará la curación habitual de la herida con técnica aséptica, verificándose la viabilidad y el edema muscular. Previo a la cobertura con apósitos, se procede a tensionar los elásticos realizando nuevos nudos sobre el extremo previamente anudado, de manera de aproximar gradual y progresivamente los bordes cutáneos. Este procedimiento se realiza también con técnica aséptica, en la cama del paciente y con mínimas molestias asociadas (Figura 2).

La tracción de la piel puede ajustarse con frecuencia diaria o cada 48 horas dependiendo del grado de disminución del edema muscular, manteniéndose así una tracción constante hasta su cierre total, lo que habitualmente ocurre alrededor de 8 a 10 días después de la intervención, con la colocación adicional de puntos con anestesia local ${ }^{7}$.

\section{Comentarios}

El aprendizaje de esta técnica data de mi período de formación como Cirujano Vascular y Endovascular en Estados Unidos en la Washington University de Saint Louis. Posteriormente la he aplicado en Chile y la he enseñado a becados vasculares. Tanto mi experiencia como la de ellos ha sido satisfactoria, lo que me ha estimulado a difundirla en este apartado.

Esta técnica está ampliamente indicada en la mayor parte de las fasciotomías, aunque deben realizarse modificaciones si se trata de fasciotomías en los muslos ${ }^{8}$. Se sugiere no utilizar esta técnica en situaciones de alto riesgo de progresión de infección (fracturas expuestas, fasceitis necrotizante, etc) ${ }^{7}$.

Comparada con otras técnicas alternativas ${ }^{9,10}$, esta modalidad de cierre es de fácil realización, bajo costo en insumos, rápido aprendizaje y corto tiempo de ejecución. Su realización ofrece ventajas de una más rápida recuperación, disminución de la morbilidad asociada a la herida, menor necesidad de re-intervenciones, menor estada hospitalaria y, finalmente, mejores resultados estéticos?

En conclusión, esta técnica de cierre diferido conocida como "shoelace technique" es de fácil ejecución y debería ser considerada como alternativa de primera elección para el manejo de fasciotomías.

\section{Referencias}

1. Tiwari A, Haq AI, Myint F, Hamilton G. Acute compartment syndromes. Br J Surg 2002; 89: 397412.

2. Velmahos GC, Theodorou D, Demetriades D, Chan L, Berne TV, Asensio J, et al. Complications and nonclosure rates of fasciotomy for trauma and related risk factors. World J Surg 1997; 21: 247-252.

3. Johnson SB, Weaver FA, Yellin AE, Kelly R, Bauer M. Clinical results of decompressive dermotomy-fasciotomy. Am J Surg 1992; 164: 286-290.

4. Rutgers PH, Van der Harst E, Koumans RK. Surgical implications of drug-induced rhabdomyolysis. Br J Surg 1991; 78: 490-492.

5. Harris I. Gradual closure of fasciotomy wounds using a vessel loop shoelace. Injury 1993; 24: 565-566.

6. Berman SS, Schilling JD, Mcintyre KE, Hunter GC, Bernhard VM. Shoelace technique for delayed primary closure of fasciotomies. Am J Surg 1994; 167: 435436.

7. Zorrilla P, Marín A, Gómez LA, Salido JA. Shoelace technique for gradual closure of fasciotomy wounds. J Trauma 2005; 59: 1515-1517.

8. Galois L, Pauchot J, Pfeffer F, Kermarrec I, Traversari R, Mainard D, et al. Modified shoelace technique for delayed primary closure of the thigh after acute compartment syndrome. Acta Orthop Belg 2002; 68: 63-67.

9. Harrah J, Gates R, Carl J, Harrah JD. A simpler, less expensive technique for delayed primary closure of fasciotomies. Am J Surg 2000; 180: 55-57.

10. Sawant MR, Hallet JP. The paper-clip modification to the vessel loop "shoelace" technique for delayed primary closure of fasciotomies. Injury 2001; 32: 619620. 